\title{
Anestesia congênita de córnea: relatos de casos
}

\section{Congenital corneal anesthesia: case reports}

Flávia Pelinsari lana ${ }^{1}$, Renato Correa Souza de Oliveira ${ }^{1}$, Ana Carolina Cabreira Vieira ${ }^{1}$, Maria Emília Xavier Santos Araújo²

\section{RESUMO}

Descrição de nove casos de anestesia congênita de córnea, sendo que desses, seis apresentavam alterações sistêmicas associadas ao quadro ocular. Três pacientes realizaram eletroneuromiografia, um sem alteração ao exame e dois com alteração isolada do ramo oftálmico do nervo trigêmeo bilateralmente. Dois pacientes tinham acuidade visual inicial melhor que 20/60 no início da avaliação e seis tinham acuidade visual final melhor que 20/60 na última visita. Todos foram submetidos a algum tipo de tratamento cirúrgico e evoluíram com opacidades corneana de tamanho variável. O tratamento dos pacientes com anestesia congênita de córnea deve ser realizado o mais precoce possível e de forma rigorosa a fim de evitar danos à transparência corneana. Investigação sistêmica, acompanhamento de perto e preparação familiar para tratamento a longo prazo e multidisciplinar são necessários para preservar a saúde ocular.

Descritores: Nervo trigêmeo/anormalidades;Doenças do nervo trigêmeo/congênito: Doenças da córnea/congênito; Relatos de casos

\section{ABSTRACT}

Case series of nine patients with congenital corneal anesthesia, six of them showed systemic changes in association with the ocular status. Three patients were submitted to electromyography, two showed isolated bilateral ophthalmic ramus alteration. Two patients had initial visual acuity better than 20/60 and six had final best corrected visual acuity better than 20/60 at the last visit. All of them were treated surgically and developed cornea opacities of variable sizes. Treatment of corneal congenital anesthesia must be performed as soon as possible to avoid corneal opacification. Systemic investigation, close follow-up and preparing the family for longterm and multidisciplinary approach are crucial to maintain the ocular health.

Keywords: Trigeminal nerve/abnormalities; Trigeminal nerve diseases/congenital; Corneal diseases/congenital; Case reports

\section{INTRODUÇÃO}

Anestesia congênita de córnea (ACC) é uma entidade rara, frequentemente mal diagnosticada e conduzida e que pode acarretar graves sequelas aos pacientes. Trata-se de um diagnóstico de exclusão que requer do oftalmologista um alto índice de suspeição e medidas terapêuticas rápidas e precisas para um bom prognóstico visual desses pacientes. Pode ser uni ou bilateral, associada ou não a anomalias sistêmicas (1,2) $^{(12}$.

A inervação sensorial da córnea é feita pela divisão oftálmica do nervo trigêmeo de onde se ramificam os nervos ciliares longos e curtos que após penetrarem no globo ocular, dirigem-se anteriormente, pelo espaço entre esclera e coroide e na altura do corpo ciliar, dando origem a cerca de 60 a 80 nervos que penetram radialmente na córnea nas posições das 3 e 9 horas ao nível dos dois terços anteriores do estroma ${ }^{(3)}$.

Além de servirem como receptores sensoriais, os nervos corneanos também têm uma função trófica e estimulam a cicatrização epitelial. Os pacientes com diminuição da sensibilidade corneana apresentam maior suscetibilidade a microtraumas mecânicos e maior fragilidade do epitélio corneano, o que pode ocasionar erosões recorrentes, úlceras neurotróficas, úlceras infecciosas secundárias, opacidades e até perfurações. O concorrente déficit do filme lacrimal piora o prognóstico desses pacientes ${ }^{(2)}$.
No presente estudo, relatamos uma série de casos de anestesia congênita de córnea, modo de apresentação, associação com doenças sistêmicas e conduta clínica e/ou cirúrgica. Foram revisados os prontuários dos pacientes com diagnóstico de anestesia congênita de córnea, atendidos no Setor de Doenças Externas Oculares e Córnea da Universidade Federal de São Paulo (UNIFESP).

\section{RELATOS DOS CASOS}

As características dos pacientes estão apresentadas na tabela 1. Dois pacientes eram filhos de pais consanguíneos (pacientes 3 e 6), e dois tinham história familiar de alteração ocular sugestiva de anestesia congênita (pacientes 2 e 4). Dois pacientes apresentavam história de automutilação na face, na região inervacional do nervo trigêmeo (pacientes 3 e 2), e um, com síndrome anestésica generalizada (paciente 3), apresentava lesões autoprovocadas por todo o corpo.

Seis pacientes apresentavam alterações sistêmicas associadas ao quadro ocular: síndrome de Gómez-López-Hernández ${ }^{(4)}$ (displasia dérmica cerebelo-trigeminal) (paciente 8), déficit do desenvolvimento neuropsicomotor atribuído ao uso de lisoprostol pela mãe durante a gestação (paciente 5), má formação do tronco encefálico e cerebelo de causa não determinada (paciente 2), síndrome anestésica generalizada (paciente 3), atraso do desenvolvimento psicomotor sem diagnóstico definido apesar dos exames neurológicos e cito-
Financiamento: Não houve financiamento para este trabalho.

Divulgação de potenciais conflitos de interesse: F.P.Lana, Não; R.C.S.Oliveira, Não; A.C.C.Vieira, Não; M.E.X.S.Araújo; Não.

Endereço de correspondência: Flávia Pelinsari Lana. Rua Doutor Diogo de Faria, 1104/134 - São Paulo (SP) - 04037-003 - Brasil - E-mail: flavia_pl@yahoo.com.br

Comitê de ética: Número do CEP é 0622/07. 
genéticos realizados (paciente 7) e um paciente apresentava surdez congênita (paciente 6).

Três pacientes realizaram eletroneuromiografia. Um deles apresentou exame normal (paciente 7) e dois alteração isolada do ramo oftálmico do nervo trigêmeo bilateralmente (pacientes 1 e 4). A sensibilidade corneana foi pesquisada com fino pedaço de algodão.

Quanto ao tratamento todos os pacientes utilizaram lubrificante tópico de modo contínuo e foram submetidos a tratamento cirúrgico para prevenir dano corneal progressivo. Evoluíram com opacidades de tamanho variável e consequente prejuízo da função visual (Figura 1).

\section{DISCUSSÃO}

Anestesia congênita de córnea (ACC) é uma doença rara que pode ocorrer devido a anomalias de desenvolvimento do tronco encefálico, alterações do núcleo do nervo trigeminal ou por defeitos neurológicos focais ${ }^{(5)}$.

A classificação de Rosenberg proposta em 1984 ainda é a mais aceita. Classicamente é dividida em três categorias ${ }^{(6)}$ :

Tipo 1: anestesia isolada do nervo trigêmeo (hipoplasia do núcleo trigeminal);
Tipo 2: associada a anomalias congênitas mesenquimais;

Tipo 3: associado a anormalidades focais do sistema nervoso central (paralisia de nervos cranianos).

No primeiro grupo, ACC sem associação com doenças neurológicas ou sistêmicas, geralmente bilateral, pode acometer todas as divisões do nervo trigêmeo ou, mais comumente, apenas a divisão oftálmica, como exemplificado pelos pacientes 1 e 4 no exame de eletroneuromiografia.

No grupo 2, a ACC é associada a doenças mesenquimais diversas. Nesses pacientes, o nervo trigêmeo é geralmente acometido em todas as suas três divisões ${ }^{(7)}$. Um defeito em algum momento da embriogênese pode ser a causa. Os pacientes 3 e 8 são classificadas nessa categoria.

Já no grupo 3, a ACC ocorre sem alterações somáticas associadas, porém com alterações focais do tronco cerebral de causas variadas. Os pacientes 2 e 5, exemplos desse grupo, apresentam hipoplasia da ponte e de parte do cerebelo ao exame de ressonância magnética.

A anestesia corneana, além da perda sensitiva, ocasiona diversas outras alterações na superfície corneana. Níveis diminuídos de neurotransmissores, como acetilcolina e substância P, são impli-

Tabela 1. Características dos pacientes com anestesia congênita de córnea

\begin{tabular}{|c|c|c|c|c|c|c|c|}
\hline Pacientes & $\begin{array}{l}\text { Idade } \\
\text { (meses) }\end{array}$ & $\begin{array}{l}\text { Antecedente } \\
\text { familiar }\end{array}$ & $\begin{array}{l}\text { Anomalia } \\
\text { sistêmica }\end{array}$ & $\begin{array}{l}\text { Localização da } \\
\text { anestesia }\end{array}$ & $\begin{array}{l}\text { Úlcera de córnea } \\
\text { tratamento cirúrgico }\end{array}$ & $\begin{array}{l}\text { AV inicial } \\
\text { OD - OE }\end{array}$ & $\begin{array}{l}\text { AV final } \\
\text { OD - OE }\end{array}$ \\
\hline 1 & 64 & Não & Não & Córnea & $\begin{array}{c}\text { Unilateral tarsorrafia + oclusão } \\
\text { pontos lacrimais }\end{array}$ & $20 / 40-20 / 200$ & $20 / 30-20 / 60$ \\
\hline 2 & 28 & $\operatorname{Sim}$ & $\operatorname{Sim}$ & $\begin{array}{l}\text { Região do } \\
\text { trigêmeo }\end{array}$ & $\begin{array}{l}\text { Bilateral tarsorrafia + } \\
\text { transplante córnea }\end{array}$ & $P L-S P L$ & $P L-S P L$ \\
\hline 3 & 16 & Não & $\operatorname{Sim}$ & Córnea & $\begin{array}{l}\text { Unilateral tarsorrafia + } \\
\text { membrana amniótica }\end{array}$ & $20 / 40-20 / 60$ & $20 / 30-20 / 40$ \\
\hline 4 & 126 & $\operatorname{Sim}$ & Não & Ramo oftálmico & $\begin{array}{l}\text { Bilateral membrana amniótica } \\
+ \text { + transplante córnea }\end{array}$ & $C D-C D$ & $20 / 40-20 / 40$ \\
\hline 5 & 5 & Não & $\operatorname{sim}$ & $\begin{array}{l}\text { Região do } \\
\text { trigêmeo }\end{array}$ & $\begin{array}{c}\text { Bilateral tarsorrafia + oclusão } \\
\text { pontos lacrimais }\end{array}$ & 20/150 - PL & 20/100 - PL \\
\hline 6 & - & - & $\operatorname{Sim}$ & $\begin{array}{l}\text { Região do } \\
\text { trigêmeo }\end{array}$ & $\begin{array}{c}\text { Não tarsorrafia + oclusão } \\
\text { ponto lacrimal }\end{array}$ & $20 / 80-20 / 60$ & $20 / 80-20 / 60$ \\
\hline 7 & 50 & Não & Sim & Generalizada & $\begin{array}{c}\text { Unilateral tarsorrafia + oclusão } \\
\text { ponto lacrimal }\end{array}$ & $20 / 60-20 / 200$ & $20 / 40-20 / 60$ \\
\hline 8 & 156 & Não & $\operatorname{Sim}$ & Generalizada & Unilateral tarsorrafia & $-/-$ & $20 / 40$ \\
\hline 9 & 48 & Não & Não & Córnea & Unilateral tarsorrafia & $-/-$ & $20 / 30$ \\
\hline
\end{tabular}

$\mathrm{AV}=$ acuidade visual; $\mathrm{PL}=$ percepção luminosa; $\mathrm{SPL}=$ sem percepção luminosa; $\mathrm{CD}=$ conta dedos
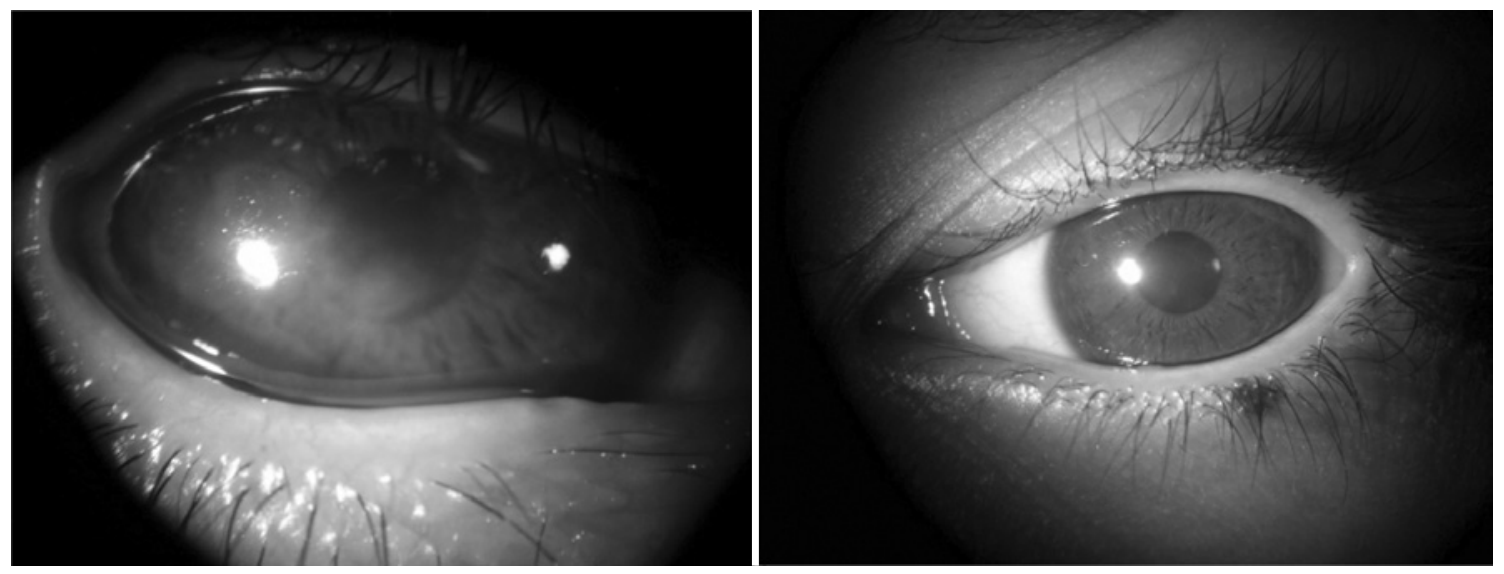

Figura 1. Olho direito com leve opacidade corneana e tarsorrafia temporal, olho esquerdo com tarsorrafia temporal. 
cados como colaboradores na disfunção epitelial e dificuldade de re-epitelização desses pacientes. Além disso, acarreta alterações do reflexo do piscar e do lacrimejamento. O resultado dessas alterações é uma grande suscetibilidade a ulcerações, defeitos epiteliais persistentes e perfurações, que podem ocasionar leucomas corneanos e comprometer a acuidade visual ${ }^{(1,2,5)}$ (Figura 1).

Depois de confirmado o diagnóstico de ACC, a sensibilidade no território inervacional do trigêmeo e a sensibilidade sistêmica devem ser pesquisadas. O uso de eletroneuromiografia pode ajudar a diferenciar entre o acometimento motor e sensorial. Um acompanhamento pediátrico e neurológico é fundamental e muita vezes somente uma avaliação genética pode definir o diagnóstico sistêmico do paciente(5)

O tratamento dos pacientes com ACC deve ser realizado o mais precoce possível e de forma rigorosa a fim de evitar danos à transparência corneana. As principais opções de tratamento incluem lubrificante tópico de forma intensiva, antibiótico tópico, lente de contato terapêutica, membrana amniótica e tarsorrafia ${ }^{(8)}$. A tarsorrafia é uma indicação recorrente por se tratar de um procedimento cirúrgico seguro e efetivo em casos de defeito epitelial persistente.

O uso do fator de crescimento neural também já foi descrito. Parece ajudar na re-epitelização mas não se mostrou eficaz na manutenção da superfície corneana sadia ${ }^{(9)}$

Os pais devem ser bem orientados por se tratar de uma condição crônica e incurável na maioria dos casos, o risco de lesões corneanas autoprovocadas deve ser alertado, orienta-se manter acompanhamento com outros especialistas já que a maioria destes pacientes apresentam manifestações sistêmicas ${ }^{(10)}$.

Baixa visão, fotofobia, injeção conjuntival e ulceração corneana na ausência de dor numa criança devem chamar a atenção do oftalmologista geral, a pesquisa da sensibilidade corneana deve ser rotineira em todos estes casos.

\section{REFERÊNCIAS}

1. Kara-José N, Belfort Jr R. Córnea: clínica e cirúrgica. São Paulo: Roca; 1996.

2. Groos EB Jr. Neurotrophic keratitis. In: Krachmer JH, Mannis MJ, Holland EJ. Cornea: cornea and external disease: clinical diagnosis and management. $2^{\text {nd }}$ ed. Elsevier Mosby; 2005. p.1189-96.

3. Garcia AP, Tarcha FAG, Vitiello Neto V, Martinelle EV, Rehder JRCL. Estesiometria corneana pós cirurgia fotorrefrativa. Rev Bras Oftalmol. 2009:68(5):271-7.

4. Zaldívar-Pascua G, Dávila-Gutiérrez G, Fernández-Álvarez H. Síndrome de GómezLópez-Hernández (displasia cerebelo-trigémino-dérmica). Informe de un caso. Acta Pediatr Mex. 2011;32(5):292-6.

5. Ramaesh K, Stokes J, Henry E, Dutton GN, Dhillon B. Congenital corneal anesthesia. Surv Ophthalmol. 2007:52(1):50-60. Review.

6. Rosenberg ML. Congenital trigeminal anaesthesia. A review and classification. Brain. 1984;107(Pt 4):1073-82.

7. Shorey P, Lobo G. Congenital corneal anaesthesia: problems in diagnosis. J Pediatr Ophthalmol Strabismus. 1990;27(3):143-7.

8. Mathen MM, Vishnu S, Prajna NV, Vijayalakshmi P, Srinivasan M. Congenital corneal anesthesia: a series of four case reports. Cornea. 2001;20(2):194-6.

9. Tan MH, Bryars J, Moore J. Use of nerve growth factor to treat congenital neurotrophic corneal ulceration. Cornea. 2006;25(3):352-5.

10. Alves M, Dias AC, Rocha EM. Dry eye in childhood: epidemiological and clinical aspects. Ocul Surf. 2008;6(1):44-51.

\title{
$38^{\circ}$ Congresso da \\ $38^{\circ}$ Congresso da Sociedade Brasileira de Retina e Vítreo
}

\section{1 a 13 de abril de 2013}

\author{
Minas Centro
}

Belo Horizonte (MG)

\section{Informações:}

Tel.: (11) 5081-7028

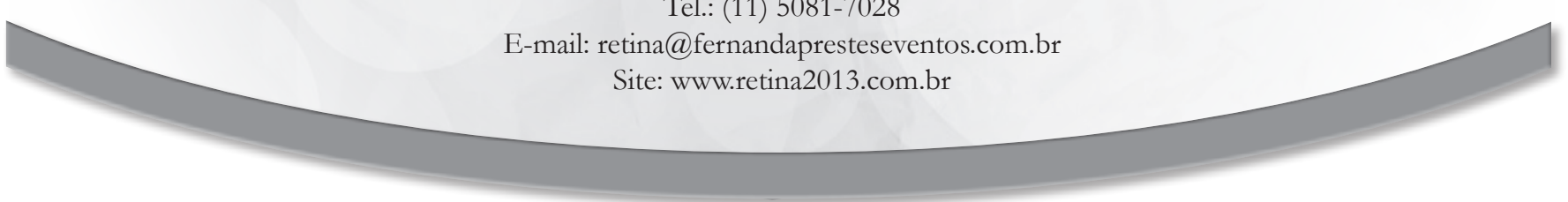

